\title{
ESTRATÉGIAS DE POSICIONAMENTO NO MERCADO INTERNACIONAL DAS EMPRESAS BRASILEIRAS DE PAPEL E CELULOSE
}

\author{
João Carlos Garzel Leodoro da Silva ${ }^{1}$, Alexandre Nascimento de Almeida ${ }^{2}$, Elisabeth Hildebrand ${ }^{3}$, \\ José Sawinski Júnior ${ }^{4}$, Pedro Giovani Lacowicz ${ }^{5}$ \\ ${ }^{1}$ Eng. Florestal, Dr., Depto. de Economia Rural e Extensão, UFPR, Curitiba, PR, Brasil - garzel@ufpr.br \\ ${ }^{2}$ Eng. Florestal, Doutorando em Ciências Florestais, UFPR, Curitiba, PR, Brasil - alexfloresta@ pop.com.br \\ ${ }^{3}$ Eng $^{\mathrm{a}}$ Florestal, Dr ${ }^{\mathrm{a}}$., Silviconsult Engenharia, Curitiba, PR, Brasil - sissi@ silviconsult.com.br \\ ${ }^{4}$ Eng. Florestal, M.Sc., Curitiba, PR, Brasil. \\ ${ }^{5}$ Eng. Florestal, Dr., Curitiba, PR, Brasil \\ Recebido para publicação: 11/04/2008 - Aceito para publicação: 24/03/2010
}

\begin{abstract}
Resumo
Este trabalho apresenta o posicionamento das empresas brasileiras exportadoras de papel e celulose em relação aos seus concorrentes. As estratégias referem-se a produto, preço, promoção, distribuição e variáveis estratégicas de marketing, para os períodos de passado e futuro. Com base no volume produzido, volume exportado e participação das exportações na produção, as empresas foram separadas em dois grupos: EGI (empresas com grande interesse no mercado externo) e EPI (empresas com pequeno interesse no mercado externo). Foi verificado um aumento da importância no posicionamento das estratégias propostas para o período futuro, na opinião dos dois grupos de empresas (EGI e EPI). Também se destaca a maior importância atribuída pelas EGI em relação ao posicionamento dessas estratégias e a importância atribuída às estratégias de produto, para as EGI e EPI, e as de preço para as EPI para os dois períodos. As estratégias de marketing estão em um nível intermediário, podendo ser consideradas, de maneira geral, como menos importantes que as estratégias de preço e produto e mais importantes que as estratégias de promoção e distribuição. As EGI no período futuro consideram as estratégias de marketing como segunda mais importante, ficando atrás apenas das estratégias de produto. Palavras-chave: Posicionamento; marketing internacional; papel e celulose; estratégias.
\end{abstract}

\begin{abstract}
Positioning strategies in international markets for Brazilian's pulp and paper companies. This work shows the Brazilian paper and pulp export companies position in relation of their outside competitors. The marketing strategies are related to product, price, promotion, distribution and other strategic variables, for the past and future periods. On the basis of the produced volume, exported volume and exported participation in the production, the companies were classified in two groups: EGI (companies with large interest in the external market) and EPI (companies with small interest in the external market). The results show an increase of the positioning importance of the strategies proposed for the future period, in the two groups opinion (EGI and EPI). Three other points are evidenced by the results as well: first, the main importance conferred to the positioning of the strategies by EGI; second, the importance conferred to the product strategies by EGI and EPI; and finally the importance conferred to the price strategies by EPI for the two periods. The marketing strategies are in an intermediate level. In general way this strategies can be considered less important than the price and product strategies and more important than the promotion and distribution strategies. The EGI companies believe the product strategies were the first more important and the marketing strategies the second one for the future period.

Keywords: Positioning; international marketing; pulp and paper; strategies.
\end{abstract}

\section{INTRODUÇÃO}

Dentro da atual fase da administração das empresas do setor de papel e celulose, tornou-se de fundamental importância para o sucesso no mercado internacional a elaboração de estratégias que auxiliem na tomada de decisões, analisando-se tanto as barreiras como os incentivos para aumentar a 
competitividade das empresas nacionais no mercado internacional, explorando um grupo de estratégias que possibilitem alcançar esse objetivo. Em se tratando de estratégias de marketing, o grupo de maior destaque deve se sobressair ao de menor, a fim de que a empresa não se exponha ao risco de perda de mercados.

A exploração do grupo de estratégias que evidencia a qualidade do produto nacional se destaca como ponto de referência a favor das empresas brasileiras, podendo concorrer ainda em preço e marketing.

Perante todas as ferramentas de marketing utilizadas pelas empresas de papel e celulose, cada empresa do setor apresenta ou explora certo grupo de estratégias, com o objetivo de manter ou ganhar mercado, dependendo das suas metas ou do tipo de mercado a ser alcançado (internacional).

$\mathrm{O}$ interesse pelas estratégias dentro das organizações está além do fiel cumprimento de orçamentos previamente projetados. Devido à significativa evolução nos últimos anos do setor de papel e celulose, torna-se claro incrementar a aplicação de estratégias de marketing, com o objetivo de manutenção ou aumento de participação de mercado, por meio de diferentes grupos de estratégias para diferentes tipos de mercados.

Uma vez que as empresas comprometem-se com suas aspirações em relação ao mercado internacional, a análise e aplicação de estratégias é fator indispensável aos administradores. Analisando fatores influentes internos e externos, a alta administração das empresas pode variar o grau de importância de cada grupo de estratégias.

Visto a importância de implementação e/ou melhoria dos processos de marketing, o presente trabalho procura verificar o posicionamento empresarial das empresas brasileiras exportadoras de papel e celulose com relação aos 4 P's (produto, preço, promoção, praça), bem como aos fatores referentes ao nível estratégico, para os períodos passado e futuro, comparando os dois períodos.

\section{REVISÃO BIBLIOGRÁFICA}

\section{$O$ setor de papel e celulose}

São poucas as pesquisas de estratégias de marketing internacional dentro do setor florestal. Em termos de Brasil, os trabalhos que mais se aproximam são os de Gimenez (1990), Da Rocha; Christensen; Cunha (1990), Azevedo (1988) e D'Angelo (1987). Esses trabalhos procuraram verificar variáveis que influenciavam o desempenho da atividade de exportação das empresas moveleiras brasileiras.

Dos trabalhos no âmbito internacional, os mais conhecidos e citados em literatura são aqueles que analisam as barreiras e incentivos para a atuação no mercado internacional (SILVA, 1996).

Sullivan; Bauerschmidt (1988; 1989) analisaram os incentivos e as barreiras dentro da indústria europeia e norte-americana de produtos florestais.

O Brasil, como praticamente toda a América Latina, sofreu, nas décadas passadas, principalmente nas de 50 e 60, uma influência elevada do pensamento econômico desenvolvido pela Comissão Econômica para América Latina e Caribe (CEPAL). A força motriz desse pensamento estava baseada na concepção do desenvolvimento de uma a política de substituição de produtos de importação, com a consequente autossuficiência (SILVA, 1996).

Dentro da ótica da política de substituição de importações, um dos setores eleitos foi o setor de papel e celulose. O setor realizou investimentos equivalentes a US\$ 6,1 bilhões entre 1989 e 1993, com projetos em execução no valor de US\$ 1,5 bilhão e outros em estudo que poderiam representar investimentos de US\$ 4,4 bilhões até o final do século, estando todos na dependência da melhoria dos preços internacionais do papel e da celulose, da estabilidade econômica do país e de financiamentos de longo prazo.

\section{Estratégias de marketing internacional}

Estratégias internacionais efetivas devem ser baseadas em vantagens competitivas (GHOSHAL, 1987). Uma vez que as estratégias são formuladas, elas devem ser implementadas pela alta administração, para que a corporação mova-se até o ponto desejado.

As estratégias são influenciadas pelo ambiente externo, indústria, natureza dos competidores e variáveis organizacionais (KOTLER, 1993). Uma das correntes de estudo de marketing e negócios internacionais tem focado os fatores que influenciam as decisões para exportar ou os processos 
envolvidos com o mercado externo, porém o processo de formulação de estratégias não tem sido exaustivamente estudado (SILVA, 1996).

Esses estudos têm examinado características de tomada de decisão, como, por exemplo, a orientação internacional, ou têm focado as vantagens competitivas da empresa. Poucos investigam a importância da relação entre vantagens competitivas e estratégia. Adicionalmente, existem poucos estudos empíricos que separam os efeitos das várias dimensões da vantagem competitiva dentro de um contexto de marketing internacional (GOMEZ; MEJIA, 1988).

Um dos primeiros estudos relacionado com fatores de sucesso nas exportações foi conduzido por Hunt; Forggatt; Howell (1967), que examinaram políticas de preço, propaganda, distribuição, crédito e serviços. As variáveis de marketing estratégico utilizadas no estudo de Koh (1991) foram operacionalizadas em termos dos 4 P's da empresa: produto no mercado externo, promoção, preço e distribuição ou ponto de venda.

Riesenbeck; Freeling (1991), Ghoshal (1987), Levitt (1983), Walters (1983), Wills; Samli; Jacobs (1991), Quelch; Hoff (1986), Wind (1986) e Szymanski, Bharadwaj; Varadarajan (1993) analisaram as estratégias de estandardização do composto de marketing entre mercados diferentes versus a adaptação para mercados individuais, focando o grau desejável de estandardização (ou adaptação) com respeito às várias estratégias competitivas, tais como marca, promoção, vendas e preço.

Há dois grupos de determinantes internos que afetam a estratégia de marketing internacional (BILKEY, 1978): (a) orientação internacional da alta administração e (b) nível de recursos compromissados pela empresa com o mercado externo. Buatsi (1980) verificou que os administradores com impressões positivas de marketing internacional respondiam melhor às estratégias para explorar as oportunidades do mercado externo.

\section{Estratégia de produto}

Vários trabalhos analisaram as vantagens da estratégia de produto com desempenho das exportações. Koh (1991) hipotetizou que uma empresa inclinada a modificar o seu produto para satisfazer as necessidades de seus consumidores teria um melhor desempenho.

A lucratividade das exportações esteve positivamente associada com a exclusividade, nos trabalhos de Khan (1978) e McGuinness; Little (1981). Porém, para Bilkey (1982), essa mesma relação não se mostrou significativa no mercado interno.

Já o nível de exportações apresentou-se positivamente relacionado com (a) exclusividade (BILKEY, 1982; McGUINNESS; LITTLE, 1981; KHAN, 1978), (b) grau de desenvolvimento de novos produtos (ROSSON; FORD, 1982) e (c) grau de serviços pré e pós-vendas (HIRSCH, 1971; CUNNINGHAM; SPIEGEL, 1971).

Entretanto essa mesma variável apresentou uma associação negativa com (a) serviços pré e pósvendas (KHAN, 1978) e (b) extensão da linha de produtos (HIRSCH, 1971; KIRPALANI; MACINTOSH, 1980). Por outro lado, o grau de adaptação dos produtos mostrou resultados conflitantes (ROSSON; FORD, 1982).

Para Cunningham; Spiegel (1971), além do serviço pré e pós-vendas, os fatores mais importantes que contribuíram para o sucesso em exportações foram design e qualidade dos produtos. Porém, para Hirsch (1971) e Kirpalani; Macintosh (1980), a extensão da linha de produtos mostrou-se negativamente correlacionada com o sucesso nas exportações.

Finalmente, Khan (1978) e McGuinness; Little (1981) sugerem que novos produtos tendem a ter maiores vantagens no mercado externo.

\section{Estratégia de preço}

Koh (1991) examinou o nível dos preços de exportação, a moeda, o método utilizado para a definição dos preços no mercado externo e os termos de avaliação do preço de exportação. Concluiu que uma empresa tem melhor desempenho quando, na venda dos produtos no mercado externo, o preço é relativamente maior do que um similar vendido no mercado doméstico, se esse preço estiver baseado na realidade do mercado externo (não somente ajustando o preço interno a um custo-base) e se adotado o preço CIF (cost, insurance and freight).

Vários estudos encontraram associações significativas do preço com o desempenho de exportações, como cita Lee (1987). Khan (1978) verificou que a proporção do preço externo em relação 
ao preço doméstico foi negativamente correlacionada com o nível de exportação, enquanto para Bilkey (1982) essa variável foi positivamente correlacionada com a lucratividade relativa das exportações. Competitividade de preço foi positivamente correlacionada com o sucesso das exportações, segundo Fenwick; Amine (1979).

Cunningham; Spiegel (1971) consideram os preços especiais como de grande influência para o desempenho das exportações, enquanto para Koh e Robicheaux (1988) o nível de preço teve uma relação significativa com a lucratividade.

\section{Estratégia de promoção}

Exportadores podem promover seus produtos através de (a) vendas pessoais, (b) promoção de vendas, (c) propaganda e (d) publicidade (SAMIEE, 1982).

Promoção é especialmente importante para empresas de países em desenvolvimento, por causa de sua imagem negativa associada com seu país de origem (PAPADOPOULUS; HESLOP; GRABY, 1988; BILKEY; NES, 1982).

As visitas pessoais aos mercados-alvos foram o fator mais importante para o sucesso de empresas britânicas, segundo Cunningham e Spiegel (1971). Já Piercy (1981) verificou que, enquanto preço foi o fator mais importante, visitas pessoais e propaganda foram os menos importantes.

O grau de propaganda foi positivamente correlacionado, segundo Tookey (1964), com o nível de exportação, enquanto o grau de esforço promocional teve influência positiva sobre o sucesso das exportações no estudo de Kirpalani; Macintosh (1980). Porém o grau de uso de propaganda não teve correlação significativa com o desempenho de exportação.

Estratégias de distribuição

Koh; Robicheaux (1988) encontraram relações significativas entre a lucratividade e canais de exportação.

Para Bilkey (1982), a exportação através de distribuidores foi percebida como mais lucrativa para bens de consumo, ao passo que exportação direta para consumidores finais foi percebida como sendo mais lucrativa para bens industriais.

Rosson; Ford (1982) verificaram que a intensidade do contato e de recursos com distribuidores, definida como sendo o nível de contatos e a troca de recurso entre exportadores e distribuidores, foi positivamente correlacionada com aquela variável (especificamente, nível das exportações). Também o grau de tomada de decisão conjunta e a concordância formal com os distribuidores foram positivamente correlacionados.

\section{MATERIAL E MÉTODO}

\section{Dados da pesquisa}

Para definição do universo de trabalho, utilizou-se o banco de dados da Associação Brasileira de Celulose e Papel (BRACELPA).

Os dados foram coletados com base em questionário dividido em dois períodos: passado (a partir de fatos ocorridos) e futuro (período não realizado ou expectativa futura). Esses questionários foram encaminhados às altas administrações de 55 empresas fabricantes e ao mesmo tempo exportadoras de papel e/ou celulose, com um retorno de $36,4 \%$ devidamente preenchidos.

Os dados coletados foram divididos em dois pontos, que caracterizam o período de cinco anos no passado (período realizado, a partir de fatos ocorridos), e perspectiva para os próximos cinco anos (período não realizado ou expectativa futura).

O questionário foi elaborado com características do tipo "estruturado não disfarçado", sendo o que melhor atende aos objetivos propostos, tanto no que tange ao levantamento de dados quanto pela área internacional das empresas.

\section{Caracterização das empresas}

Os agrupamentos foram realizados com base no seguinte modelo:

$$
\mathrm{PTE}=\mathrm{PVP}+\mathrm{PVE}+\mathrm{PPE}
$$


Em que: PTE = peso total da empresa;

$\mathrm{PVP}=$ peso do volume produzido;

$\mathrm{PVE}=$ peso do volume exportado;

$\mathrm{PPE}=$ peso do produto nas exportações da empresa.

Ainda em relação ao modelo, tem-se que:

- Se o VP (volume produzido) da empresa está 1 desvio (1s) abaixo da média, o peso atribuído no modelo é 1;

- Se VP está entre $-1 \mathrm{~s} \mathrm{e}+1 \mathrm{~s}$, o peso atribuído no modelo é 2;

- Se VP está 1s acima da média, o peso atribuído no modelo é 3.

O mesmo cálculo foi realizado para VE (volume exportado) e PE (produto exportado).

Com esse modelo, procura-se não beneficiar uma única variável na definição das empresas em relação aos mercados internacionais.

As empresas que se encontram com o peso total acima da média foram definidas como:

- EGI (Empresas com Grande Interesse no mercado externo), ou primeiro grupo; e

- EPI (Empresas com Pequeno Interesse no mercado externo), ou segundo grupo.

\section{Variáveis do trabalho}

As variáveis utilizadas no presente trabalho são do conjunto de 12 estratégias de mercado apresentado à alta administração das empresas exportadoras de papel e celulose, que permitem verificar seu posicionamento em relação aos seus concorrentes.

Essas variáveis foram subdivididas em 5 grupos de estratégias relacionadas em marketing: a) produto, b) preço, c) promoção, d) distribuição (todas estas definidas como instrumental operacional em marketing, ou seja, o marketing mix) e e) marketing estratégico.

\section{Instrumental estatístico}

Para o presente trabalho, utilizou-se análise paramétrica (média) e não-paramétrica (frequência).

As técnicas da estatística não-paramétrica são particularmente adaptáveis aos dados das ciências do comportamento. A aplicação dessas técnicas não exige suposições quanto à distribuição da população da qual se tenha retirado as amostras para análise. Podem ser aplicadas a dados que se disponham simplesmente em ordem, ou mesmo para estudo de variáveis nominais. Os testes não-paramétricos são adequados para análises de dados qualitativos.

Para a avaliação da opinião qualitativa, considerou-se a técnica do "Escalograma de Likert", que consiste em dar um peso numérico a essa opinião, variando num intervalo de 1 (nenhuma importância) a 5 (total importância). De acordo com Chisnall (1980) e Infante (1984), essa escala permite que os respondentes tenham certa liberdade para exprimir sua opinião, informando seu grau de sentimento.

Posteriormente, os dados foram analisados em termos de média aritmética simples e frequência percentual. Cada resposta representa uma média dos últimos cinco anos, evitando com isso que estejam baseadas em fatores conjunturais que possam ter afetado ou estar afetando a empresa. O mesmo ocorreu quando foi perguntado sobre a percepção futura em relação aos mesmos.

Para a análise das médias, a posição desejada foi classificada em 5 níveis: (1) muito pior, (2) pior, (3) igual, (4) melhor e (5) muito melhor.

Para a análise de frequência, ou seja, percentual de empresas que atribuem determinada posição para o conjunto de estratégias, foram considerados os seguintes intervalos: 1 a 2 (muito pior a pior), 2,01 a 3 (pior a igual), 3,01 a 4 (igual a melhor) e 4,01 a 5 (melhor a muito melhor).

\section{RESULTADOS E DISCUSSÃO}

Neste item é discutido o posicionamento das empresas brasileiras exportadoras de papel e celulose para os dois períodos (passado e futuro), para o conjunto geral de estratégias e para cada conjunto particular de estratégias (produto, preço, promoção, praça e marketing estratégico). Também são apresentados os resultados divididos entre empresas de grande interesse no mercado externo (EGI) e empresas de pequeno interesse no mercado externo (EPI). 


\section{Posicionamento global das estratégias}

A tabela 1 apresenta os resultados médios quanto ao posicionamento desejado pelas empresas em relação ao conjunto de estratégias propostas.

Assim, as EGI, nos períodos de passado e futuro, desejavam um posicionamento estratégico, respectivamente, de 3,16 e 3,54, ou seja, de igual a melhor para o conjunto como um todo, não significando, por consequência, uma mudança significativa em seu modelo estratégico, indicando uma possível satisfação com o modelo já existente.

Porém, para as EPI, houve também um aumento no posicionamento desejado do conjunto de estratégias de um período para outro, sendo que passou de 2,66 (entre pior e igual) para 3,04 (entre igual a melhor) em relação aos seus concorrentes.

Assim sendo, apesar de a maior importância ser dada pelas EGI, para o conjunto de estratégias, as EPI também apresentam um significativo aumento no grau desejado em relação ao posicionamento das estratégias de um período para o outro.

Tabela 1. Grau médio de importância para o conjunto das estratégias.

Table 1. Average degree of importance for the group of the strategies.

\begin{tabular}{lll}
\hline Período & EGI & EPI \\
\hline Passado & 3,16 & 2,66 \\
Futuro & 3,54 & 3,04 \\
\hline
\end{tabular}

Detalhando esse resultado, a tabela 2 apresenta o percentual de empresas que atribuem determinada posição para o conjunto das estratégias, de acordo com os intervalos definidos no instrumental estatístico. Dessa forma, verifica-se um aumento do grau de importância atribuído para o posicionamento das estratégias no período Futuro, em relação ao período anterior, tanto para as EGI quanto para as EPI.

Para o primeiro grupo, a posição atribuída às estratégias ficou entre 2,01 (pior) e 5 (muito melhor) nos dois períodos. O que se verifica é que houve um deslocamento das empresas em se concentrarem para o futuro em um posicionamento de igualar e melhorar em relação aos seus concorrentes as estratégias efetuadas. Significa que no passado existiam $45,5 \%$ de empresas que consideraram que suas estratégias estavam sendo piores do que as da concorrência, e com isso pretendiam mudá-las para, pelo menos, igualá-las. Esse fato mostra um reconhecimento de fraqueza estratégica e desejo de corrigi-la, o que é fundamental para a manutenção da competitividade empresarial.

Ao mesmo tempo, o segundo grupo também mostrou esse deslocamento, porém não de modo tão elevado como para as EGI, ou seja, um avanço sim, porém de forma mais modesta. Visto ser esse grupo o daquelas empresas com pequeno interesse no mercado interno, é um resultado coerente, pois toda mudança em estratégias significa a necessidade de alocação de recursos (humanos, físicos, monetários e outros) para a sua consecução, e é razoável supor o não-desejo de investir em um mercado que não é o de maior importância para eles.

Tabela 2. Importância das estratégias nos dois períodos analisados (\%).

Table 2. Importance of strategies in two analyzed periods (\%).

\begin{tabular}{lcccccccc}
\hline \multirow{2}{*}{ Período } & \multicolumn{4}{c}{ EGI } & \multicolumn{4}{c}{ EPI } \\
\cline { 2 - 9 } & $\mathbf{1 ~ a ~ 2 ~}$ & $\mathbf{2 , 0 1} \mathbf{a} 3$ & $\mathbf{3 , 0 1}$ a 4 & $\mathbf{4 , 0 1} \mathbf{a} \mathbf{5}$ & $\mathbf{1 ~ a ~ 2}$ & $\mathbf{2 , 0 1}$ a 3 & $\mathbf{3 , 0 1} \mathbf{a} 4$ & $\mathbf{4 , 0 1}$ a 5 \\
\hline Passado & 0,0 & 45,45 & 45,45 & 9,09 & 28,57 & 28,57 & 42,86 & 0,0 \\
Futuro & 0,0 & 9,09 & 72,73 & 18,18 & 12,50 & 37,50 & 50,00 & 0,0 \\
\hline
\end{tabular}

\section{Posicionamento das estratégias de produto}

Analisando apenas o posicionamento das estratégias referentes ao $\mathrm{p}$ de produtos do marketing mix, observa-se que, tanto para as EGI quanto para as EPI, ocorre um aumento da importância dada a essas estratégias no período futuro, em relação ao período anterior.

Para as empresas do primeiro grupo, o posicionamento permaneceu entre o nível 2,01 (pior) e 5 (muito melhor) nos dois períodos, porém repetindo o resultado de concentração das empresas no grupo de igual a melhor, enquanto que para as empresas do segundo grupo houve um deslocamento para as classes superiores de forma relativamente homogênea (Tabela 3). 
Tabela 3. Importância das estratégias de produto nos dois períodos analisados (\%).

Table 3. Product strategies importance in the two analyzed periods (\%).

\begin{tabular}{lcccccccc}
\hline \multirow{2}{*}{ Período } & \multicolumn{9}{c}{ EGI } & \multicolumn{4}{c}{ EPI } \\
\cline { 2 - 9 } & $\mathbf{1}$ a 2 & $\mathbf{2 , 0 1}$ a 3 & $\mathbf{3 , 0 1}$ a 4 & $\mathbf{4 , 0 1} \mathbf{a ~ 5}$ & $\mathbf{1 ~ a ~ 2}$ & $\mathbf{2 , 0 1} \mathbf{a} 3$ & $\mathbf{3 , 0 1}$ a 4 & $\mathbf{4 , 0 1}$ a 5 \\
\hline Passado & 0,00 & 45,25 & 36,36 & 18,18 & 14,29 & 42,86 & 42,86 & 0,00 \\
Futuro & 0,00 & 9,09 & 72,73 & 18,18 & 0,00 & 37,50 & 50,00 & 12,50 \\
\hline
\end{tabular}

Portanto o grau de posicionamento médio atribuído pelas EGI, nos períodos de passado e futuro, passou de 3,45 a 3,80, ou seja, igual a melhor para as estratégias de produto. Já para as EPI, o grau de posicionamento passou de 2,86 (entre pior a igual) para 3,34 (igual a melhor), de acordo com a tabela 4.

Apesar de o posicionamento médio em importância ter aumentado mais para as empresas do segundo grupo, de um período para outro, os resultados da tabela 3 mostram claramente que as empresas do primeiro grupo ainda atribuem maior importância a esse tipo de estratégia.

Tabela 4. Média do posicionamento para as estratégias de produto.

Table 4. Average of positioning product strategies.

\begin{tabular}{lll}
\hline Período & EGI & EPI \\
\hline Passado & 3,45 & 2,86 \\
Futuro & 3,80 & 3,34 \\
\hline
\end{tabular}

Posicionamento das estratégias de preço

Também em relação ao posicionamento das estratégias de preço, tanto para as empresas do primeiro grupo como para as do segundo, houve um aumento do nível de percepção da importância dada a essas estratégias no período futuro.

Assim, conforme resultados das tabelas 5 e 6, observa-se que as EPI apresentam uma preocupação maior quanto ao posicionamento das estratégias de preço do que as EGI. Isso mostra que essas estratégias constituem fator muito importante para as empresas com pequeno interesse no mercado externo, ao passo que, para as empresas com grande interesse no mercado externo, apesar de existir, logicamente, uma preocupação, ela é percebida de maneira mais moderada.

Analisando esse aspecto com base nas frequências das respostas, chega-se à mesma conclusão, com alguns detalhes interessantes referentes à transição de um período para outro.

Para as EGI, no período passado, a maior frequência de respostas, $81,80 \%$, foi obtida para o nível 2,01 (pior) a 3 (igual), contra 57,14\% das EPI para o mesmo período, indicando, ainda, com 14,29\% de respostas, o nível 3,01 (igual) a 4 (melhor). Observa-se que as empresas com grande interesse no mercado externo indicaram que seu posicionamento não alcançava esse nível de qualidade, apontando percepções diferentes que podem indicar a visão de mercado, dada sua dependência (Tabela 5).

Para o período futuro (posicionamento desejado), verifica-se um deslocamento nos dois grupos para uma melhoria em relação aos concorrentes, o que implicará estratégias diferenciadas das realizadas no passado.

Pode-se verificar que apenas $27,3 \%$ das EGI consideravam o posicionamento das estratégias futuras de preço entre 3,01 (igual) a 4 (melhor) aos concorrentes, ao passo que para as EPI esse percentual foi de $50,0 \%$.

Tabela 5. Percentual do posicionamento atribuído às estratégias de preço.

Table 5. Positioning percentage attributed to price strategies.

\begin{tabular}{lcccccccc}
\hline \multirow{2}{*}{ Período } & \multicolumn{4}{c}{ EGI } & \multicolumn{4}{c}{ EPI } \\
\cline { 2 - 10 } & $\mathbf{1 ~ a ~ 2 ~}$ & $\mathbf{2 , 0 1}$ a 3 & $\mathbf{3 , 0 1}$ a 4 & $\mathbf{4 , 0 1}$ a 5 & $\mathbf{1 ~ a ~ 2}$ & $\mathbf{2 , 0 1}$ a 3 & $\mathbf{3 , 0 1}$ a 4 & $\mathbf{4 , 0 1}$ a 5 \\
\hline Passado & 18,18 & 81,20 & 0,00 & 0,00 & 28,57 & 57,14 & 14,29 & 0,00 \\
Futuro & 18,18 & 54,55 & 27,27 & 0,00 & 12,50 & 37,50 & 50,00 & 0,00 \\
\hline
\end{tabular}

O grau de posicionamento médio atribuído pelas EGI, nos períodos passado e futuro, passou de 2,82 para 3,09, ou seja, igual a melhor para as estratégias de preço. Já para as EPI, o grau de importância passou de 2,86 (entre pior a igual) para 3,38 (igual a melhor), mudança maior do que a do primeiro grupo 
de empresas.

Assim sendo, observa-se uma vez mais que no período passado havia uma grande concordância com respeito aos níveis de percepção das empresas dos dois grupos. Porém, para o período futuro, houve uma preocupação maior por parte das EPI em objetivarem alcançar um melhor posicionamento do que as empresas com grande interesse no mercado externo (Tabela 6).

Tabela 6. Média do posicionamento para as estratégias de preço.

Table 6. Positioning average for the price strategies.

\begin{tabular}{lll}
\hline Período & EGI & EPI \\
\hline Passado & 2,82 & 2,86 \\
Futuro & 3,09 & 3,38 \\
\hline
\end{tabular}

\section{Posicionamento das estratégias de promoção}

Quanto ao posicionamento das estratégias de promoção, os dois grupos de empresas (EGI e EPI) atribuem um aumento da importância dessas estratégias para o futuro quando comparado com o passado.

Apesar das EPI apresentarem um maior aumento no grau de posicionamento médio dessas estratégias de um período para outro, as EGI atribuem maior importância para essas estratégias nos dois períodos, conforme resultados das tabelas 7 e 8 .

O grupo de empresas com grande interesse no mercado interno indicou um posicionamento igual aos concorrentes no período passado, como continuam, no seu conjunto, neste patamar no período futuro. $\mathrm{O}$ que se verifica é que aquelas que indicavam posicionamento pior no passado iriam pelo menos se igualar no futuro com os concorrentes. Ao mesmo tempo, 9,09\% das empresas desse grupo iriam procurar um melhor posicionamento em promoção do que os concorrentes, o que serve como indicativo de mudança de percepção da importância desses instrumentos para a competitividade empresarial (Tabela 7).

Tabela 7. Percentual do posicionamento atribuído às estratégias de promoção.

Table 7. Positioning percentage attributed to promotion strategies.

\begin{tabular}{lcccccccc}
\hline \multirow{2}{*}{ Período } & \multicolumn{9}{c}{ EGI } & \multicolumn{4}{c}{ EPI } \\
\cline { 2 - 9 } & $\mathbf{1}$ a 2 & $\mathbf{2 , 0 1}$ a 3 & $\mathbf{3 , 0 1}$ a 4 & $\mathbf{4 , 0 1}$ a 5 & $\mathbf{1 ~ a ~ 2}$ & $\mathbf{2 , 0 1}$ a 3 & $\mathbf{3 , 0 1}$ a 4 & $\mathbf{4 , 0 1}$ a 5 \\
\hline Passado & 36,36 & 63,64 & 0,00 & 0,00 & 28,57 & 42,86 & 14,20 & 14,29 \\
Futuro & 0,00 & 90,91 & 9,09 & 0,00 & 25,00 & 0,00 & 50,00 & 25,00 \\
\hline
\end{tabular}

Para as EPI, o grau perceptivo de posicionamento mostrou-se mais diluído, no período passado, do que o indicado pelas empresas do primeiro grupo, indicando menor consenso entre essas empresas, ou diferentes estratégias adotadas no passado, que as colocaram em diferentes posições frente aos concorrentes. Porém existiu também uma concentração, menor do que para as empresas do primeiro grupo, no nível de igualdade de posicionamento com os concorrentes. Também para esse grupo houve um deslocamento para um desejo de estar melhor posicionado em estratégias promocionais do que os concorrentes (Tabela 7).

O grau de posicionamento médio atribuído pelas EGI, nos períodos de passado e futuro, para as estratégias de promoção, passou de 2,64 a 3,09, ou seja, pior a igual. Já para as EPI, o grau de posicionamento passou de 2,14 (pior a igual) para 2,75, permanecendo no mesmo nível (Tabela 8).

Tabela 8. Média do posicionamento para as estratégias de promoção.

Table 8. Average of the positioning for the promotion strategies.

\begin{tabular}{lll}
\hline Período & EGI & EPI \\
\hline Passado & 2,64 & 2,14 \\
Futuro & 3,09 & 2,75 \\
\hline
\end{tabular}

Assim, pode-se observar que o grau de posicionamento médio das EGI com relação às estratégias de promoção passou de um nível pior para igual, enquanto as EPI permaneceram no mesmo nível (pior), apesar de um significativo aumento entre o percebido e o desejado.

Esses resultados demonstram que as EPI estão atentas ao posicionamento das estratégias de 
promoção com relação aos seus concorrentes, porém existe uma parcela considerável de empresas que desconsideram totalmente as estratégias de promoção, o que pode ser observado pelos percentuais do nível 1 a 2 na tabela 7.

\section{Posicionamento das estratégias de distribuição}

Os dois grupos de empresas (EGI e EPI) atribuem um aumento no grau de posicionamento das estratégias de distribuição para o período futuro em relação ao período passado.

As EGI atribuem maior aumento, nos dois períodos considerados, de acordo com os resultados apresentados nas tabelas 9 e 10, em que o grau de posicionamento permaneceu entre o nível 2,01 (pior) e 4 (melhor), em passado, e entre o nível 2,01 (pior) e 5 (muito melhor), em futuro.

Em passado, 54,5\% das EGI consideravam as estratégias de distribuição como entre pior a igual, e 45,6\% como igual a melhor. Para o período de futuro, 72,7\% passaram a considerar como igual a melhor, e 9,1\% como melhor a muito melhor (Tabela 9).

Para as EPI, o grau de posicionamento permaneceu entre o nível 1 (muito pior) e 4 (melhor), nos dois períodos. Entretanto, em passado, $42,9 \%$ das EPI atribuíam grau muito pior a pior às estratégias de promoção, 14,3\% entre pior a igual e 42,9\% achavam igual a melhor. Em futuro, 50,0\% atribuíam grau muito pior a pior a essas estratégias, e 50,0\% achavam igual a melhor (Tabela 9).

Tabela 9. Percentual do posicionamento atribuído às estratégias de distribuição.

Table 9. Positioning percentage attributed to the distribution strategies.

\begin{tabular}{lcccccccc}
\hline \multirow{2}{*}{ Período } & \multicolumn{9}{c}{ EGI } & \multicolumn{4}{c}{ EPI } \\
\cline { 2 - 9 } & $\mathbf{1 ~ a ~ 2}$ & $\mathbf{2 , 0 1}$ a 3 & $\mathbf{3 , 0 1}$ a 4 & $\mathbf{4 , 0 1}$ a 5 & $\mathbf{1 ~ a ~ 2}$ & $\mathbf{2 , 0 1}$ a 3 & $\mathbf{3 , 0 1}$ a 4 & $\mathbf{4 , 0 1}$ a 5 \\
\hline Passado & 0,00 & 54,55 & 45,55 & 0,00 & 42,86 & 14,29 & 42,86 & 0,00 \\
Futuro & 0,00 & 18,18 & 72,73 & 9,09 & 50,00 & 0,00 & 50,00 & 0,00 \\
\hline
\end{tabular}

O grau de importância médio atribuído pelas EGI, nos períodos de passado e futuro, passou de 3,07 a 3,40, ou seja, igual a melhor para o posicionamento das estratégias de distribuição. Já para as EPI, o grau de importância passou de 2,65 (entre pior a igual) para 2,72 (Tabela 10).

Com base nesses resultados, observa-se que para o posicionamento das estratégias de distribuição as EGI dão importância maior do que as EPI.

Tabela 10. Média do posicionamento para as estratégias de distribuição.

Table 10. Average of the positioning for the distribution strategies.

\begin{tabular}{lll}
\hline Período & EGI & EPI \\
\hline Passado & 3,07 & 2,65 \\
Futuro & 3,40 & 2,72 \\
\hline
\end{tabular}

\section{Posicionamento das estratégias de marketing}

Analisando apenas as estratégias de marketing, conclui-se que, tanto para as EGI quanto para as EPI, houve um aumento considerável no nível de percepção do posicionamento dado a essas estratégias no período futuro em relação ao período anterior.

O posicionamento médio em importância aumentou de maneira muito semelhante para os dois grupos de empresas, porém as EGI dão importância maior do que as EPI para o período passado. Para o período futuro, as EGI continuam com um grau de posicionamento maior que as EPI para essas estratégias (Tabelas 11 e 12).

Para as EGI, o grau de posicionamento permaneceu entre os níveis 1 (muito pior) e 4 (melhor) para o período passado, e entre os níveis 2,01 (pior) a 5 (muito melhor) para o período futuro. Em passado, 54,5\% das EGI consideravam as estratégias de marketing como pior a igual, e 18,2\% como igual a melhor. Para o período de futuro, 45,5\% passaram a considerar essas estratégias como igual a melhor, e 9,1\% como melhor a muito melhor (Tabela 11).

Para as EPI, o grau de posicionamento permaneceu entre o nível 1 (muito pior) e 5 (muito melhor) em passado, e entre o nível 2,01 (pior) e 5 (muito melhor) para o período futuro. Verificando o período passado, $14,3 \%$ das EPI atribuíam grau muito pior a pior às estratégias de marketing, $57,1 \%$ consideravam pior a igual e 28,6\% melhor a muito melhor. Em futuro, 25,1\% atribuíam grau pior a igual 
a essas estratégias, $37,6 \%$ igual a melhor e 37,6\% melhor a muito melhor (Tabela 11).

Tabela 11. Percentual do posicionamento atribuído às estratégias de marketing.

Table 11. Percentile of the positioning attributed to the marketing strategies.

\begin{tabular}{lcccccccc}
\hline \multirow{2}{*}{ Período } & \multicolumn{9}{c}{ EGI } & \multicolumn{1}{c}{ EPI } \\
\cline { 2 - 10 } & $\mathbf{1}$ a 2 & $\mathbf{2 , 0 1}$ a 3 & $\mathbf{3 , 0 1}$ a 4 & $\mathbf{4 , 0 1} \mathbf{a ~ 5}$ & $\mathbf{1 ~ a ~ 2}$ & $\mathbf{2 , 0 1}$ a 3 & $\mathbf{3 , 0 1}$ a 4 & $\mathbf{4 , 0 1}$ a 5 \\
\cline { 2 - 10 } Passado & 27,27 & 54,55 & 18,18 & 0,00 & 14,29 & 57,14 & 0,00 & 28,57 \\
Futuro & 0,00 & 45,45 & 45,45 & 9,09 & 0,09 & 25,09 & 37,59 & 37,59 \\
\hline
\end{tabular}

O grau de posicionamento médio atribuído pelas EGI nos períodos de passado e futuro passou de 2,91 para 3,64, ou seja, atribuiu-se grau igual a melhor às estratégias de marketing. Já para as EPI, o grau de importância passou de 2,43 (pior a igual) para 3,13 (igual a melhor), de acordo com a tabela 12.

Tabela 12. Média do posicionamento para as estratégias de marketing.

Table 12. Average of the positioning for the marketing strategies.

\begin{tabular}{lll}
\hline Período & EGI & EPI \\
\hline Passado & 2,91 & 2,43 \\
Futuro & 3,64 & 3,13 \\
\hline
\end{tabular}

\section{CONCLUSÕES}

- Dados os resultados encontrados, pode-se concluir que as altas administrações das empresas de papel e celulose, no global, mudaram seu modelo de relacionamento com o mercado internacional, dandolhe maior importância.

- Essa conclusão baseia-se justamente no grau de importância que elas atribuíram às estratégias para aquele mercado.

- Isso permite inferir que elas entendem a importância e um provável aumento de concorrência existente e, com isso, necessitam incrementar suas estratégias em relação aos concorrentes para, pelo menos, manterem a participação que já detêm.

- Do marketing mix, para as empresas com maior interesse nesse mercado, as estratégias de produto, seguidas de distribuição, são as mais importantes, podendo-se inferir incremento de qualidade e de eficiência na distribuição.

- Porém, para as empresas com pouco interesse naquele mercado, preço é mais importante, seguido de produto, de onde se pode inferir a procura por aumento de participação naquele mercado com preços menores, sendo que as estratégias de produto devem ser realizadas permitindo o alcance desse objetivo.

- Apesar de o posicionamento médio em importância ter aumentado mais para as EPI, de um período para outro, os resultados da tabela 4 mostram claramente que as EGI ainda atribuem maior importância a esse tipo de estratégia.

- Já as EPI, em passado, consideravam as estratégias de preço e produto como sendo as mais importantes, seguidas das de distribuição e marketing. Para o período subseqüente, passaram a considerar como mais importante as estratégias de preço, depois produto, a seguir marketing e por fim promoção.

- Fica assim evidenciada a importância atribuída às estratégias de produto, para as EGI e EPI, e as de preço para as EPI para os dois períodos. As estratégias de marketing estão em um nível intermediário, podendo ser consideradas, de maneira geral, como menos importante que as estratégias de preço e produto, e mais importantes que as estratégias de promoção e distribuição. As EGI no período futuro consideram as estratégias de marketing como segunda mais importante.

\section{REFERÊNCIAS}

AZEVEDO, C. Q. M. O uso de métodos de exportação e o controle do composto de marketing na indústria brasileira de móveis. Rio de Janeiro: COPPEAD/UFRJ. 1988, Dissertação MBA.

BILKEY, W. J. An attempted integration of the literature on the export behavior of firms. Journal of International Business Studies. v. 9, n. 1, p. 33-46, 1978. 
. Variables associated with export profitability. Journal of International Busines Studies: v. 13, n. 3, p. 57-72, 1982.

BILKEY, W. J.; NES, E. Country-of-origin effects for uni-national and bi-national products. Journal of International Business Studies. (Summer): 89-99, 1982.

BUATSI, S. N. The influence of organizational factors on the export potential and export growth of firms. Ph.D. dissertation, University of Manchester. 1980.

CHISNALL, P. Pesquisa mercadológica. São Paulo, Saraiva, 1980.

CUNNINGHAM, M. T.; SPIEGEL, R. I. A study in successful exporting. British Journal of Marketing. p. 2-12, 1971.

D'ANGELO F. L. A cooperação para exportação na indústria de móveis. Rio de Janeiro: COPPEAD/UFRJ. 1987. Dissertação MBA.

DA ROCHA, A.; CHRISTENSEN, C.; CUNHA, C. E. Aggressive and passive exporters: a study in the Brazilian furniture industry. International Marketing Review, v. 7, n. 5, p. 6-15, 1990.

FENWICK, I.; AMINE, L. Export performance and export policy: evidence from UK clothing industry. Journal of the Operational Research Society. v. 30, n. 8, p. 747 - 754. 1979.

GHOSHAL, S. Global strategy: on organization framework. Strategic Management Journal. 8(September/October): 425-40, 1987.

GIMENEZ, F. A. P. Comportamento estratégico na pequena indústria moveleira. Revista de Administração, v. 25, n. 2, p. 3-11, 1990.

GOMEZ-MEJIA, L. R. The role of human resources strategy in export performance: a longitudinal study. Strategic Management Journal. v. 9. p. 493-505. 1988.

HIRSCH, S. The export performance of six manufacturing industries. New York: Praeger, 1971.

HUNT, G. G.; FROGGATT, J. D.; HOWELL, P. J. The management of export marketing in engineering industries. British Journal of Marketing. v. 1 (Spring), p. 10-24, 1967.

INFANTE, V. S. Aspectos dos problemas de marketing na experiência de exportação através de consórcios de pequenas e médias empresas (um estudo no estado de São Paulo). Universidade de São Paulo, 1984. 282 p. Tese.

QUELCH, J. A.; HOFF, E. J. Customizing global marketing. Harvard Business Review. (May/June): p. 59-68. 1986.

KHAN, M. S. A study of success a failure in exports. Stockholm: Akademilitteraur, 1978.

KIRPALANI, V. H.; MACINTOSH, N. B. International marketing effectiveness of technology-oriented small firm. Journal of International Business Studies. p. 81-90, 1980.

$\mathrm{KOH}$, A. C. Relationships among organizational characteristics marketing strategy and export performance. International Marketing Review, v. 8, n. 3, p. 46-60, 1991.

$\mathrm{KOH}$, A. C.; ROBICHEAUX, R. A. Variations in export performance due to differences in export marketing strategy: implications for industrial markets. Journal of Business Research, v. 17, n. 3, p. 294-258, 1988.

KOTLER, P. Administração de marketing. São Paulo: Atlas, 1993. 848 p.

LEE, C. H. Export market expansion strategies and export performance: a study of high technology manufacturing firms. University of Washington, 1987. 272 p. Tese.

LEVITT, T. The globalization of markets. Harvard Business Review. 61 (May/June): p. 92-102, 1983.

McGUINNESS, N. W.; LITTLE, B. The influence of product characteristics on export performance of new industrial products. Journal of Marketing, p. 110-122, 1981. 
PAPADOPOULUS, N. G.; HESLOP, L. A.; GRABY, F. Does “country-of-origin" matter? Some findings from a cross-cultural study of consumer views about foreign products. Marketing Science Institute, Report n. 87-104. 1988.

PIERCY, N. Company internationalization: active and reactive exporters. European Journal of Marketing. v. 13, n. 3, p. 26-40. 1981.

RIESENBECK, H.; FREELING, A. How global are global brands? The McKinsey Quarterly, v. 4, p. 318, 1991.

ROSSON, P. J.; FORD, D. Manufacturer-overseas distributor relations and export performance. Journal of International Business Studies, v. 13, n. 3, p. 57-72, 1982.

SAMIEE, S. Elements of marketing strategy a comparative study of US and Non-US based companies. Journal of International Business Studies, (Spring/Summer): p. 119-26, 1982.

SZYMANSKI, D. M.; BHARADWAJ, S. G.; VARADARAJAN, P. R. Standardization versus adaptation of international marketing strategy: an empirical investigation. Journal of Marketing, v. 57 (October), p. 1-17, 1993.

SILVA, J. C. G. L. da. Análise da formulação de estratégias de marketing internacional de empresas de papel e celulose. Piracicaba, 1996. 247 p. Tese (Doutorado em Economia Aplicada) - Escola Superior de Agricultura Luiz de Queiroz, Universidade de São Paulo.

SULLIVAN, D.; BAUERSCHMIDT, A. Common factors underlying barriers to export: a comparative study in the European and U.S. Paper Industry. Marketing International Review, v. 29, n. 2, p. 17-32, 1989.

TOOKEY, A. Factors associated eith success in exporting. Journal of Management Studies, March. p. 48-66, 1964.

WALTERS, P. G. P. Export information sources: a study of their usage and utility. International Marketing Review, 11 (Winter): p. 33-43, 1983.

WILLS, J.; SAMLI, A. C.; JACOBS, L. Developing global products and marketing strategies: a construct and research agenda. Journal of Academy Marketing Science, 19 (Winter): p. 1-10, 1991.

WIND, Y. The myth of globalization. Journal of Consumer Marketing, 3 (Spring): p. 23-6, 1986. 